\title{
Philonsorbonne
}

11 | 2017

Année 2016-2017

\section{Faiblesse cognitive et faiblesse morale chez les stoïciens}

Olivier D'JERANIAN

\section{OpenEdition}

1 Journals

Édition électronique

URL : https://journals.openedition.org/philonsorbonne/908

DOI : 10.4000/philonsorbonne.908

ISSN : 2270-7336

Éditeur

Publications de la Sorbonne

Édition imprimée

Date de publication : 1 janvier 2017

Pagination : 173-193

ISSN : 1255-183X

\section{Référence électronique}

Olivier D'JERANIAN, « Faiblesse cognitive et faiblesse morale chez les stoïciens », Philonsorbonne [En ligne], 11 | 2017, mis en ligne le 05 janvier 2017, consulté le 21 septembre 2021. URL : http:// journals.openedition.org/philonsorbonne/908; DOI : https://doi.org/10.4000/philonsorbonne.908

(c) Tous droits réservés 


\title{
Faiblesse cognitive et faiblesse morale chez les stoïciens
}

\author{
Olivier D'JERANIAN \\ (Université Paris 1 Panthéon-Sorbonne - UMR 7219 GRAMATA)
}

« À défaut de nos actes, Nos frayeurs feront de nous des traîtres ».

(Macbeth, Acte IV, scène 2)

\section{L'interprétation comme mesure de l'action}

Lorsqu'Arrien rapporte ce que dit Épictète de l'infanticide commis par Médée, il attribue à ce dernier une formule qui n'est pas sans parodier Protagoras. Pour le philosophe de Nicopolis, en effet, « ce qui apparait, pour

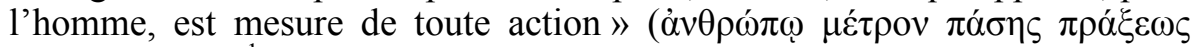

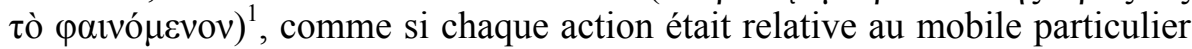
apparaissant à l'agent, dans la mesure où c'est bien « ce qui semble le plus avantageux » qui, une fois validé comme tel, met ce dernier en mouvement ${ }^{2}$. Ce qui rend intégralement raison de l'acte tragique de Médée, c'est son caractère avantageux, que celle-ci aurait à la fois interprété, vu et suivi, comme si rien ne la déterminait extérieurement à agir ainsi.

En tant que détermination de l'agir, l'apparaître (le palvó $\mu \varepsilon v o v)$ est donc une interprétation qui est à la fois l'origine pratique de l'action et la règle morale de l'agent. Epictète suggère que Médée a jugé bon d'agir ainsi de son propre point de vue, et qu'elle se serait aperçue de l'erreur de cette perspective si elle avait considéré correctement la situation.

S'opposant au relativisme protagoréen, l'intellectualisme moral stoïcien suppose que la mesure phénoménologique de l'action sera dite "juste»

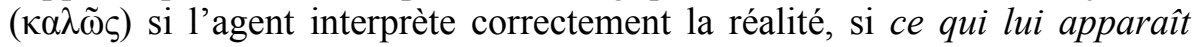

\footnotetext{
1. Épictète, Entretiens I, 28, 13-14 (désormais E pour les Entretiens et $M$ pour le Manuel).

2. Id., 1-6.
} 
est conforme à ce qui est. Dans ce cas, l'individu sera "irréprochable »

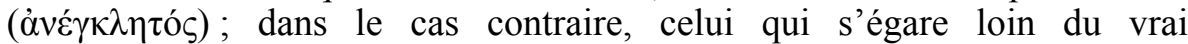
(ó $\pi \varepsilon \pi \lambda \alpha \nu \eta \mu \varepsilon ́ v o \varsigma$ ) en subira seul le dommage. Cela signifie que le « $\varphi \alpha \imath{ }^{\prime} \mu \varepsilon v o v »$ décrit, pour Épictète, une activité spécifiquement humaine et néanmoins propre à chaque homme, une vision normative qui implique une reprise active, dans le jeu interprétatif, de l'agent. Cette lecture permet au stoïcien d'en faire le critère pour juger de l'état d'une âme saine ou viciée, appréhendable dans le comportement qu'il mesure, déterminant en retour la responsabilité de l'agent vis-à-vis de ses actions et de ses propres états psychiques.

En nous servant de la métaphore théâtrale souvent utilisée par Épictète pour illustrer la condition humaine ${ }^{3}$, on pourrait dire que l'être humain est comme un acteur et que l'interprétation de son rôle dépend entièrement de la compréhension de celui-ci, qu'il doit saisir comme une matière, un thème qu'il n'a pas le pouvoir de changer, pour bien le jouer.

Cette analogie trouve chez Épictète une traduction technique, qui engage la psychologie stoïcienne. La distinction entre le thème théâtral et son jeu se retrouve dans la différence que le stoïcien suggère entre la $\varphi \alpha v \tau \alpha \sigma i ́ \alpha$ (l'impression), généralement conçue comme involontaire et ne dépendant pas de l'homme, et le paıvó $\mu \varepsilon v o v$ (l'apparaissant), qui dépend de l'homme et qui possède un caractère foncièrement pratique car dogmatique.

En ajoutant le $\varphi \alpha$ vó́ $\mu \varepsilon v o v$ à la distinction déjà canonique entre

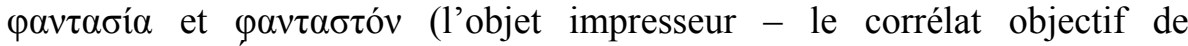
l'impression), Epictète voudrait-il expliciter le double aspect de l'impression, à la fois passive et active, c'est-à-dire, toujours déjà re-présentation ?

Si toute représentation est une interprétation, c'est parce qu'elle engage nécessairement, à cause de la nature rationnelle de l'âme sur laquelle s'imprime l'objet extérieur, un point de vue particulier sur celui-ci. Cette mise en perspective est essentiellement critique avant d'être ou de ne pas être pratique, puisqu'elle suppose la combinaison de deux jugements librement produits et validés par l'agent: un jugement "thétique » (de type il y $a X$ ) et un jugement de type "axiologique » (de type s'il y a X, alors il convient de faire $Y$ ) initiant et réglementant une impulsion et une action immédiatement à venir (l'action Y). On comprend à présent l'intérêt de la métaphore théâtrale, l'interprète jouant son rôle à la mesure de sa compréhension des intentions que l'auteur de la pièce avait eues lorsqu'il l'a écrit.

Mais c'est aussi dire que, pour être pratique, l'apparition du mobile à l'agent doit être réductible à des composants purement dogmatiques. Reste à savoir comment, dans l'idée d'une "représentation pratique», s'articulent la $\varphi \alpha v \tau \alpha \sigma i ́ \alpha$ et le $\varphi \alpha i v o ́ \mu \varepsilon v o v$. Cela revient à comprendre comment, au sein même de l'âme, Épictète nous invite à penser - à la suite

3. Cf. ibid. I, 29, 37-43; M. XVII. 
de Chrysippe $^{4}$ - l'union synthétique de ce qui ne dépend pas de nous (l'impression, c'est-à-dire la représentation dans son versant passif) et ce qui dépend de nous (l'interprétation, c'est-à-dire la représentation dans son versant actif). Nous verrons que le paıvó $\mu \varepsilon v o v$ peut d'abord se comprendre, en régime stoïcien, comme une modification dogmatique de la $\varphi \alpha v \tau \alpha \sigma i ́ \alpha$. Il sera ainsi défini comme une opinion $(\delta o ́ \gamma \mu \alpha)$ visible, ou plutôt une vision normative pour l'action humaine. Ce point nous permettra de comprendre que la faiblesse morale est avant tout une faiblesse cognitive.

\section{Impression et interprétation : le processus de la représentation}

L'invention par Zénon du concept de $\varphi \alpha v \tau \alpha \sigma i ́ \alpha$, fondé sur le jeu réflexif de la lumière $(\varphi \tilde{\omega} \varsigma)$ - à la fois éclairante et visible - décrit le lien primitif que l'homme entretient avec le monde. Passive, l'âme humaine subit ses impressions, les reçoit comme de l'extérieur par son contact avec un objet impresseur ${ }^{5}$. Mais dans sa dimension active, il faudrait plutôt dire que l'âme humaine se représente toujours des objets (réels ou non), et que ces représentations ne peuvent être que rationnelles ou techniques en vertu de la nature rationnelle de son âme. Dès lors que ces impressions sont nécessairement reprises « in animo », c'est-à-dire, dès qu'au choc psychologique une réponse de l'âme est donnée par l'assentiment validant notamment un jugement de valeur apposé à un jugement thétique, la représentation devient mesurée par son récepteur, et peut engager une action.

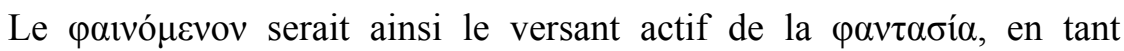
qu'elle est déjà une interprétation propre à l'agent, c'est-à-dire en tant qu'elle est grevée d'une densité dogmatique relative à la nature de l'âme de

\footnotetext{
4. Cicéron, dans le De Fato ( $\$ 43)$, nous indique que, pour Chrysippe, l'élan provoqué par la $\varphi \alpha v \tau \alpha \sigma i \alpha$, qui ne dépend pas de nous, est une extrinsecus pulsa qui a lieu in animo; le

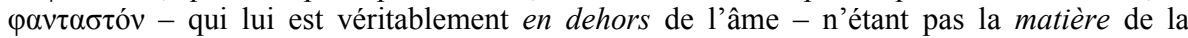
réponse mais son occasion. Par conséquent, l'extériorité de la représentation est seulement liée à son origine (un $\varphi \alpha v \tau \alpha \sigma \tau o ́ v$ ) et non à son lieu.

5. La représentation ne dépend pas de nous (contrairement à l'assentiment) et est une empreinte laissée dans l'âme (D.L. VII, 46) affectant et modifiant nécessairement cette

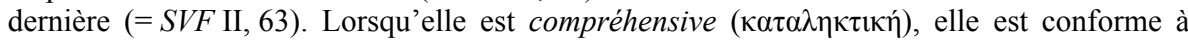
l'objet dont elle est la marque exacte. Ce point fait précisément l'objet de la controverse avec les philosophes sceptiques. Cependant, les stoïciens reconnaissent également qu'il arrive souvent que l'objet existe mais, parce que celui-ci n'est pas assez clair, la représentation n'est pas suffisamment nette pour qu'un assentiment lui soit donné. Lorsqu'elle n'est pas issue d'un objet réel (un $\varphi \alpha v \tau \alpha \sigma \tau o ́ v)$, elle est un phantasme $(\varphi \alpha ́ v \tau \alpha \sigma \mu \alpha)$ - une imagination - et son objet (irréel) prend le nom de phantastikon ( Traditionnellement, depuis Zénon, la représentation est définie comme « une affection qui se produit dans l'âme, et qui se révèle elle-même en même temps que ce qui l'a produite ». (Aëtius, IV, 12, 1-5 = SVF II. 54 ; = LS 39 B). La polémique entre Cléanthe et Chrysippe sur la nature de la représentation, tient au fonctionnement de la mémoire, compris sur la base d'une théorie plus "matérialiste» $(\varphi \alpha \nu \tau \alpha \sigma i ́ \alpha=$ impression $)$ de Cléanthe, et plus « fonctionnaliste » $(\varphi \alpha v \tau \alpha \sigma i ́ \alpha=$ représentation) de Chrysippe (D.L. VII, 50-51 = LS 39 A).
} 
l'agent. On pourrait dire que le paivó $\mu \varepsilon v o v$ est un produit psychique de synthèse, puisqu'il mélange des composants extérieurs (le mode de tension de l'objet «impresseur ») et intérieurs (la qualité de l'âme de l'agent). Cela permettrait aux stoïciens d'apporter leur solution au problème classique de la vérité comme cohérence et du critère pour juger de la bonne interprétation. En effet, il existera deux types de $\varphi \alpha i v o ́ \mu \varepsilon v \alpha$, les vrais (les cognitions) et les faux (les opinions ou simples apparences), représentant deux façons pour la $\varphi \alpha v \tau \alpha \sigma i ́ \alpha$ d'apparaître - une façon juste, qu'on dira "cognitive » ou «kataleptique » (qui «saisit» son objet), et une façon erronée, qu'on appellera « non cognitive» :

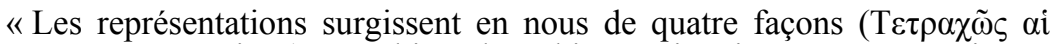

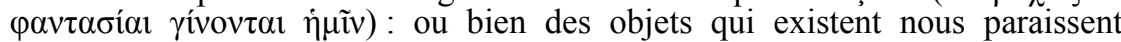

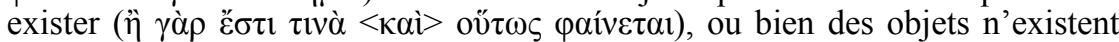

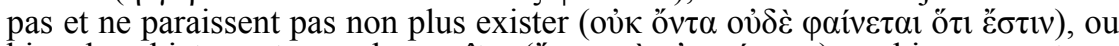

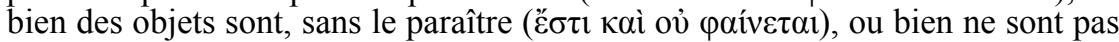

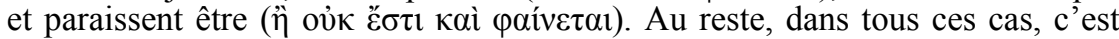
l'affaire du philosophe de tomber juste. Quelle que soit la difficulté qui nous importune, nous devons trouver contre elle le remède efficace $»^{6}$.

Il y aurait donc quatre façons d'interpréter, qui correspondent à quatre possibilités pour la chose représentée d'être ou de ne pas être (soit la chose est, soit elle n'est pas - en accord ou désaccord avec - soit elle paraît être, soit elle ne paraît pas être). Et c'est justement parce que la $\varphi \alpha v \tau \alpha \sigma \sigma^{\prime} \alpha$ apparaît

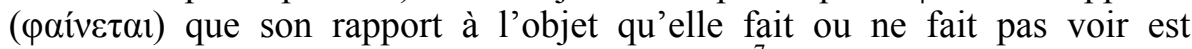
susceptible d'être authentique ou inauthentique ${ }^{7}$. On comprend maintenant pourquoi le paivó $\mu \varepsilon v o v$ devient l'enjeu de toute l'attention philosophique, en indexant le domaine de l'agir sur le domaine épistémique.

Qu'il s'agisse là, comme le dit Épictète, de la tâche même du philosophe que de faire la part correcte des choses ne doit pas nous surprendre : si le

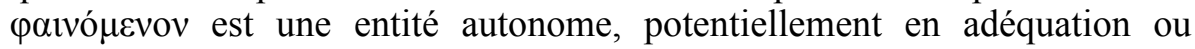
inadéquation avec la réalité, il doit être examiné attentivement parce qu'il implique des dispositions psychologiques et des états mentaux qui détermineront le bonheur ou le malheur de l'homme (c'est l'objet de la troisième discipline), mais également sa vertu ou son vice :

« Tout comme les enfants se laissent effrayer par l'apparence redoutable

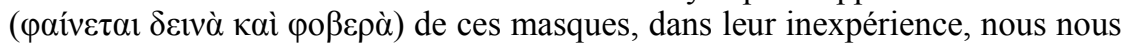
laissons nous aussi impressionner ( $\pi \alpha ́ \sigma \chi 0 \mu \varepsilon v)$ par les événements pour la même raison et de la même façon que les enfants par les épouvantails $»^{8}$.

6. Épictète, E. I, 27, 1-2 (trad. Souilhé).

7. Seule la représentation compréhensive est le critère de vérité, en tant qu'adéquation parfaite entre l'objet et son apparition. Témoignant de l'inséparabilité de la morale et de la science, elle représente, dans le champ épistémique, l'état psychologique du sage - l'objet est gravé dans l'âme en conformité avec sa réalité. Cf. Sextus Empiricus, Adv. Math. VII, 151.

8. Épictète, E. II, 1, 15 (trad. Souilhé). 
Comme Socrate cherchait à «retourner les masques »" Épictète clarifiera le lien entre la passion, comme affection et comme comportement, et l'interprétation. La distinction faite entre $\varphi \alpha$ ivó $\mu \varepsilon v o v$ et $\varphi \alpha v \tau \alpha \sigma i ́ \alpha$ est en réalité cruciale pour saisir l'enjeu de la détermination des états psychologiques de l'agent par les représentations. Comme y insiste Épictète, cette distinction est, au sens littéral, d'ordre dogmatique. Ainsi, une représentation "pénible» devra être analysée dans ses composantes phénoménologiques - il faudra, pour assurer le bonheur, en retrancher la valeur dogmatique, qui nous apparaît (comme pénible), et qui n'est l'effet que d'un mauvais usage de cette représentation, de notre libre interprétation (Marc Aurèle instituant cette pratique en règle) ${ }^{10}$. S'il faut céder devant ce qui relève de l'infirmité de notre condition humaine, l'enjeu éthique consistera à se concentrer sur l'usage critique des $\varphi \alpha \iota v o ́ \mu \varepsilon v \alpha$, en tant qu'ils sont dogmatiquement déterminés ${ }^{11}$. Cela suppose que le paivó $\mu \varepsilon v o v$, qui dépend de nous, peut être différent, l'exercice typique de la philosophie consistant à réduire la $\varphi \alpha v \tau \alpha \sigma i ́ \alpha$, quand elle est «pénible », à un $\varphi \alpha$ เvó $\mu \varepsilon v o v$ axiologiquement neutre. C'est d'ailleurs l'une des premières leçons du Manuel (I) :

«Immédiatement, pour chaque représentation pénible ( $\pi \alpha \dot{\sigma} \sigma \eta\rceil$ $\varphi \alpha v \tau \alpha \sigma i \alpha \underline{\alpha}$

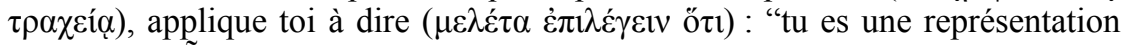

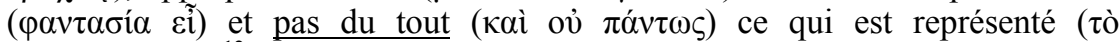
( $a$ livó $\mu \varepsilon v o v) "{ }^{12}$.

Victor Goldschmidt ${ }^{13}$ a souligné que ce passage défiait la leçon des prédécesseurs stoïciens, en réfutant la théorie de la compréhension, Épictète se lançant dans une sorte de préfiguration de l'idéalisme kantien, dans la mesure où la compréhension s'appliquerait au phénomène et non à la chose en soi (l'objet de la représentation). Ce faisant, Goldschmidt a proposé ${ }^{14}$ de traduire "ov̉ $\pi \alpha ́ v \tau \omega \varsigma$ » par l'expression "pas tout à fait», comme si c'était l'activité et non le réel qui devait faire l'objet d'une "critique». Pierre Hadot, qui remarque la fausseté de cette interprétation du Manuel ${ }^{15}$, montre qu'il s'agit plutôt d'interroger le coefficient dogmatique de la représentation, non pas quand elle est compréhensive, mais quand elle est douteuse, et surtout "pénible». Ce faisant, Hadot traduit « $\varphi \alpha v \tau \alpha \sigma i ́ \alpha »$ par « représentation subjective» ou «pure représentation», « ov $\pi \alpha ́ v \tau \omega \varsigma »$ par

9. Cf. Platon, Phédon 77e, Criton 46c (thème repris par Marc Aurèle, Pensées XI, 23).

10. Cf. Marc Aurèle, P. V, 24 ; VI, 13 ; VII, 35, 48 ; VIII, 13, 24, 49 ; IX, 32, 36 ; X, 11, 17,

18. En suivant Épictète, cf. E. I, 20, 7 ; II, 18, 24 ; III, 8 ; Manuel IV, XLIV, XLV.

11. Cf. Id., E. III, 12, 15.

12. Id., M. I, 5 (nous traduisons).

13. V. Goldschmidt, Le Système stö̈cien et l'idée de temps, Paris, Vrin, 1959, (1953), p. 120 121.

14. Id., p. 185.

15. P. Hadot, La Citadelle intérieure. Introduction aux Pensées de Marc Aurèle, Paris, Arthème Fayard, 1997 (1992), p. 189 et suiv. 
«pas du tout», et « (c'est-à-dire, l'objet réellement perçu).

Toutefois, cette nouvelle lecture ne nous permet plus de comprendre pourquoi Médée, qui n'a fait que suivre son interprétation, "s'est

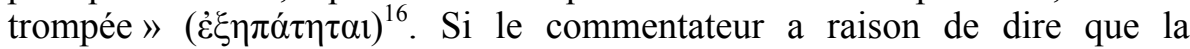
discipline philosophique consiste à interroger la valeur dogmatique de la représentation, c'est sur le $\varphi \alpha$ เó $\mu \varepsilon v o v$, en tant qu'il est précisément le résultat de la modification dogmatique de la $\varphi \alpha v \tau \alpha \sigma i \alpha$, que doit porter l'interrogation (et non sur la $\varphi \alpha v \tau \alpha \sigma i ́ \alpha)$. Faire du palvó $\mu \varepsilon v o v$ « ce qui apparaît réellement», et le privilégier par rapport à la «représentation subjective » ( $(\alpha \nu \tau \alpha \sigma i \alpha)$, nous conduirait à associer Épictète et Marc Aurèle au pyrrhonisme bien plus qu'au stoïcisme hellénistique ${ }^{17}$.

Ainsi, la $\varphi \alpha v \tau \alpha \sigma i ́ \alpha$, lorsqu'elle est, par la tension de son objet impresseur, « convaincante ${ }^{18}$ (et notamment quand elle est «pénible »), est facilement affectée d'un coefficient dogmatique négatif puissant (relativement à la faiblesse de l'âme qui la subit), qui mesurera ensuite

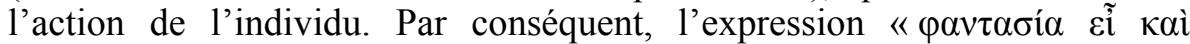

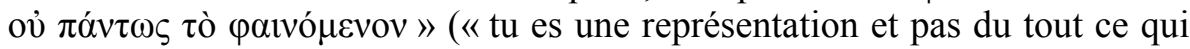
est représenté ») montre que l'examen des représentations passe par la dissociation entre, d'une part, la pénibilité réelle (due à des propriétés physiques de l'objet) de la représentation, et la pénibilité interprétée comme

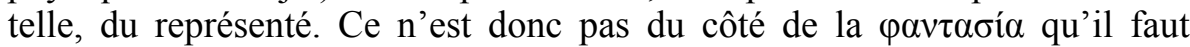
trouver la subjectivité et l'ajout dogmatique, comme le croit Hadot (qui pense qu'Épictète invite à revenir à un «pur palvó $\mu \varepsilon v o v »)$, mais bien du côté du pavvó $\mu \varepsilon v o v$, qui implique déjà la modification dogmatique. En «se répétant » que la représentation n'est que ce qu'elle est, on ne maintient pas la suspension du jugement, la mise entre parenthèse phénoménologique, mais on empêche la $\varphi \alpha v \tau \alpha \sigma i ́ \alpha$ de devenir un mauvais paıvó $\mu \varepsilon v o v$, une interprétation fautive, en lui interdisant de "déployer la série de ses tableaux $»{ }^{19}$. Cela tient au fait que, contrairement à Sextus, Épictète ne pense pas qu'il soit possible qu'un $\varphi \alpha \imath v o ́ \mu \varepsilon v o v$ soit privé de $\delta o ́ \gamma \mu \alpha$. On en trouve un exemple dans un passage qui implique par ailleurs la seule autre occurrence de l'expression « oủ $\pi$ óv $\tau \omega \varsigma$ » chez Épictète :

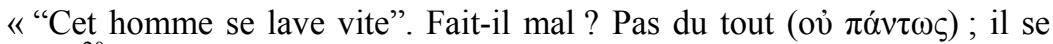
lave vite $»^{20}$.

16. Épictète, E. I, 28, 8. Il semble que Hadot confonde ici la $\varphi \alpha v \tau \alpha \sigma i ́ \alpha$ avec le $\varphi \alpha ́ v \tau \alpha \sigma \mu \alpha$ du texte aurélien de $P$. XI, 19 (certes très proche de $M$. I, 5).

17. Par exemple Sextus, Hyp. I, 21 (où le paıvó $\mu \varepsilon v o v$ est le critère de la « voie sceptique »). Voir aussi I, $138 ; 182 ; 186 ; 216 ; 219$; II, $88 ; 89 ; 91 ; 94 ; 124 ; 126$; etc.

18. Voir Épictète, E. II, 22, 6 ; Plutarque, Stoic. Rep. 1055F-1057B (=SVF II, 994 ; III, 177) ; Marc Aurèle, P. VIII, 26; 36 ; Sextus Empiricus, Adv. Math. VII, 242-244 (=SVF II, 65); Galien PHP V, 5, 19 (= Posid. fr. 416, 60).

19. Cf. Épictète, E. II, 18, 23-26.

20. Ibid. IV, 8, 2 (trad. Souilhé). Voir aussi M. XLV. 
On voit bien ici que la représentation ( $(\alpha v \tau \alpha \sigma i \alpha)$ de l'homme se lavant vite ne doit pas du tout (ov̉ $\pi \alpha ́ v \tau \omega \varsigma$ ) nous "apparaître comme » ( où Épictète explique que le trouble psychologique n'intervient pas, à

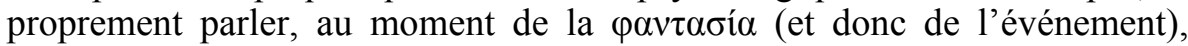
mais au moment de son interprétation :

«Ce ne sont pas les choses qui troublent les hommes, mais les opinions qu'ils en ont ; comme la mort n'est en rien terrible (puisqu'à Socrate aussi elle

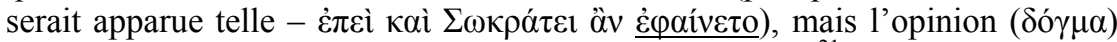
sur la mort, qu'elle est terrible, c'est là ce qui est terrible $»^{21}$.

C'est bien parce que Socrate n'a pas interprété la mort comme une chose terrible qu'il n'a pas craint de mourir, et, partant, qu'il n'a pas tenté de s'enfuir, comme un mauvais acteur jouerait mal son rôle. Au niveau psychologique, le $\delta$ ó $\gamma \mu \alpha$ sur la mort (suivant lequel la mort est terrible), prend une dimension immédiatement phénoménale pour être pratique et normative. On pourrait citer par ailleurs au moins deux autres passages dans lesquels Épictète évoque le paıvó $\mu \varepsilon v o v$ comme dogmatiquement positif, en tant qu'il est le propre d'un homme libre :

1) «Donc: dès à présent juge-toi digne de vivre en homme adulte qui

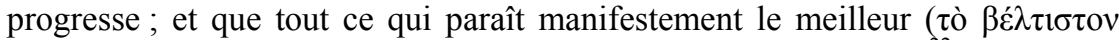
paivó $\mu \varepsilon v o v)$ soit pour toi une loi inviolable (vó

2) «Qui peut encore me faire obstacle et contrarier mon propre sentiment

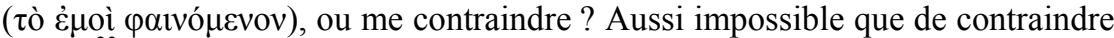
Zeus $»^{23}$.

Vraisemblablement, le pavvó $\mu \varepsilon v o v$, l'interprétation de la $\varphi \alpha v \tau \alpha \sigma i ́ \alpha$, est ce qui a la possibilité de rendre l'homme vulnérable ou invincible. Cela suppose qu'il s'impose à l'agent avec la force d'une norme morale. Il sera

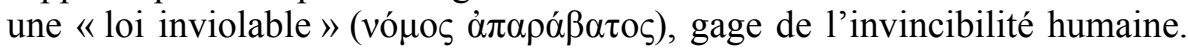
Dans la mesure où il est une production humaine mesurant l'action, il est

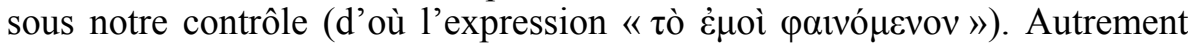
dit, le $\varphi \alpha$ wó $\mu \varepsilon v o v$ est pathogène seulement s'il est erroné :

«Quiconque a une claire conscience de ce fait que, pour l'homme, la

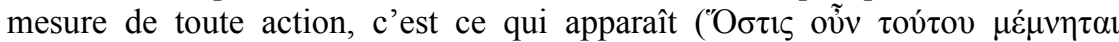

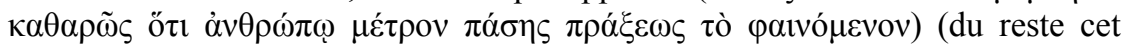

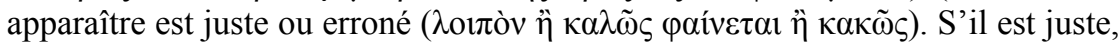
l'homme est irréprochable; s'il est erroné, il en subit lui-même la peine, car il est impossible qu'un homme se trompe et qu'un autre en éprouve le dommage), qui donc a conscience de cela ne se mettre en colère contre personne, ne

21. Nous traduisons.

22. Id. M. LI, 2 (trad. Cattin).

23. Ibid. E. IV, 1, 90 (trad. Souilhé). 
s'irritera contre personne, n'injuriera personne, ne blâmera personne, ne haïra, n'offensera personne $»^{24}$.

Différence cruciale avec le pyrrhonisme, on s'aperçoit que le paivó $\mu \varepsilon v o v$ n'est jamais axiologiquement ni dogmatiquement neutre. Avant d'illustrer cette idée avec Médée, soulignons, d'une part, que sommes entièrement responsables de nos actes en tant qu'ils sont mesurés uniquement par les opinions que nous formons nous-mêmes à l'égard des choses et des événements; d'autre part, que nous pouvons nous reconnaître dans nos actes puisque le palvó $\mu \varepsilon v o v$ est symptomatique de notre disposition psychologique ; et enfin, dans la mesure où le paivó $\mu \varepsilon v o v$ ne concerne que nous-mêmes, il ne peut atteindre ni endommager autrui.

Comme le souligne Épictète, « il est impossible qu'un homme se trompe et qu'un autre en éprouve le dommage ». Cette proposition s'explique par le fait qu'il ne peut exister un paivó $\mu \varepsilon v o v$ commun, et que chacun suit et subit ses propres interprétations. De toute évidence, puisque le paıvó $\mu \varepsilon v o v$ décrit le lieu psychologique d'apparition de notre liberté, il devient l'enjeu de l'analyse morale $e^{25}$. En tant que «mesure de toute action», qu'elle soit insignifiante ou terrible, le pavvó $\mu \varepsilon v o v$ se voit immédiatement grevé d'une valeur morale, et doit être confronté aux prénotions $(\pi \rho 0 \lambda \eta \dot{\psi \varepsilon \varepsilon \varsigma})^{26}$ :

« Voici pourquoi on ne peut s'étonner assez de la façon d'agir habituelle. Quand nous voulons juger de ce qui est pesant, nous ne jugeons pas au petit bonheur, ni non plus quand nous voulons juger de ce qui est droit ou tordu; bref, quand il nous importe de connaître la vérité dans ce domaine, nul d'entre nous ne fera jamais rien au petit bonheur. Mais s'agit-il de la principale et unique cause du succès ou de l'erreur, de la prospérité ou de l'adversité, du malheur ou de la félicité, dans ce cas seulement nous nous livrons au petit bonheur et à l'irréflexion. Nulle part ici l'équivalent d'une balance, nulle part l'équivalent d'une règle, mais quelque chose m'a paru bon et aussitôt je le fais. Suis-je donc plus fort qu'Agamemnon ou Achille pour pouvoir seul me contenter de ce qui apparaît, alors que ces derniers, pour avoir suivi ce qui apparaît, ont causé et subi de telles calamités? Et quelle tragédie a commencé autrement? L'Atrée d'Euripide, qu'est-ce que c'est? Ce qui apparait. L'Edipe de Sophocle, qu'est-ce que c'est ? Ce qui apparait. Phénix ? Ce qui apparaît. Hippolyte ? Ce qui apparaît. Quelle sorte d'homme vous paraît donc être celui qui ne fait aucun cas de tout cela ? Comment appelle-t-on ceux qui suivent la première chose qui nous apparaît ?

- Des fous

- Et nous, alors, faisons-nous autre chose ? » ${ }^{27}$

24. Ibid. I, 28, 10.

25. Ibid. III, 22, 42-44.

26. Ibid. E. II, 11, 15.

27. Ibid. E. I, 28, 28-33 (trad. Souilhé modifiée). 
Après avoir convaincu son interlocuteur qu'il est impossible de ne pas choisir ce qui nous apparaît comme un avantage ${ }^{28}$, en déterminant l'assentiment par l'avantage apparent, Épictète s'explique sur Médée, qui soutient qu'elle a été vaincue dans ses résolutions par son courroux ${ }^{29}$, et ce, bien qu'elle ait fait le pire. Si Médée se trompe, c'est parce qu'elle était aveugle à son avantage réel, mais aussi parce qu'elle n'a pas vu que la satisfaction qu'exigeait d'elle son courroux relevant déjà d'une interprétation fautive de sa part. Faible moralement parce que cognitivement, comme les aveugles ou les boiteux, ses "facultés essentielles» sont «aveuglées et mutilées $\gg{ }^{30}$ :

«- On ne peut donc trouver une chose avantageuse sans la choisir?

- On ne le peut ${ }^{31}$.

- Comment [Médée] peut-elle dire :

Oui, je sais tout le mal que je vais accomplir ;

Mais mon courroux, plus fort, a vaincu ma raison ${ }^{32}$ !

- Parce que cela même, satisfaire son courroux et se venger de son époux, elle le regarde comme plus avantageux que de sauver ses enfants

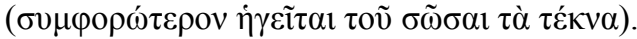

- Oui mais elle s'est trompée.

- Montre-lui clairement qu'elle s'est trompée et elle ne le fera pas $(\delta \varepsilon \tilde{\xi} \xi o v$

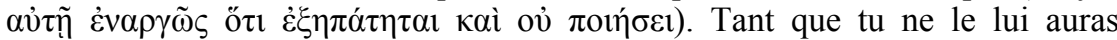

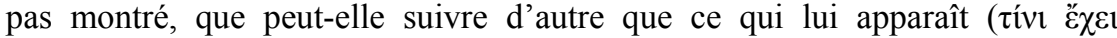

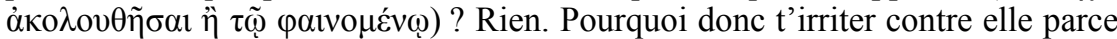
qu'elle se trompe, la malheureuse, sur les sujets les plus graves, et que, d'être humain, elle s'est transformée en vipère? Mais, s'il le faut absolument, ne dois-tu pas plutôt plaindre, comme nous plaignons les aveugles ou les boiteux, ceux dont les facultés essentielles sont aveuglées et mutilées ? (...).

- De sorte que les actions, grandes et terrifiantes, ont également cette

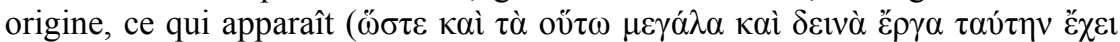

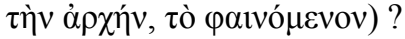

- Celle-là et pas d'autres. L'Iliade n'est rien de plus que représentation

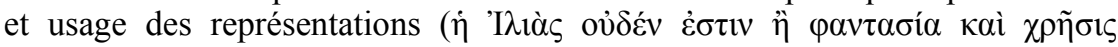

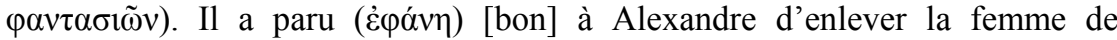
Ménélas, il a paru [bon] à Hélène de le suivre. Eh bien, s'il avait semblé [bon]

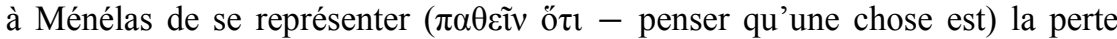
d'une telle femme comme un avantage, que serait-il arrivé ? C'en était fait de l'Iliade, et non seulement de l'Iliade, mais aussi de l'Odyssée »33.

28. Ibid. I, 28, 6-7.

29. Cf. Euripide, Médée, v. 1078-1079.

30. Épictète, E. I, 28, 9.

31. Voir id. III, 7, $15 ; 22,42$.

32. Euripide, Médée, 1078 sq.

33. Épictète, E. I, 28, 6-13. 
Épictète indexe dans ce passage le choix (de l'infanticide) sur la perception de l'avantage. Cela signifie qu'il n'est pas possible de fonder la responsabilité morale de l'agent sur une liberté de choisir, si l'on entendait en tout cas celle-ci comme un libre arbitre. Si l'interprétation est libre, sa force et son caractère pratique tiennent précisément dans son caractère subtil, où ce qui apparaît à l'agent - l'avantage apparent - est pris pour ce qui est réellement. Autrement dit, l'agent ne voit pas, au sens littéral du terme, qu'il n'a pas affaire à un donné, mais à une construction logique.

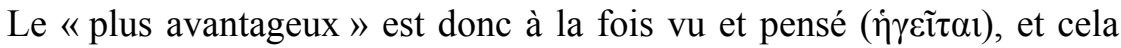
implique une considération du sujet sur sa propre identité, dans un rapport réflexif - Médée s'imagine offensée et pense que l'infanticide est la chose la plus utile à faire pour rétablir l'injustice. Comparer cette dernière à une aveugle signifie que celle-ci ne peut pas voir autre chose en la répudiation qu'une offense et en l'infanticide qu'un préférable, et donc le suivre (selon D.L. VII, 86-88) comme si c'était son bien propre. Cela signifie que le paivó $\mu \varepsilon v o v$ est le produit d'une âme défectueuse, incapable d'aller contre ses propres visions, comme si l'apparition erronée de l'avantage déterminait nécessairement et tragiquement - c'est le cas de le dire ici-l'action.

\section{Quelle excuse pour les faibles?}

Si toute faiblesse morale est cognitive, reste à savoir comment les stoïciens s'expliquent non seulement sur la responsabilité du « faible », mais aussi ce qui distingue chez lui l'incapacité d'agir (aboulie) de celle de se

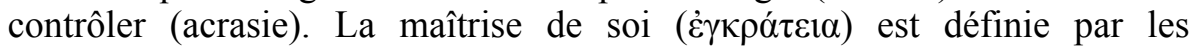
philosophes du Portique comme « une disposition qui ne saurait faillir aux prescriptions de la raison droite ou bien une manière d'être qui ne se laisse pas vaincre par les plaisirs $»^{34}$. Dans les rapports de Diogène Laërce et de Stobée, elle apparaissait comme une sous-espèce de la vertu (par opposition

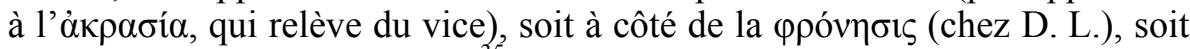
subordonnée à la $\sigma \omega \varphi \rho \sigma^{\prime} v \eta^{35}$. Cléanthe la considérait comme une force

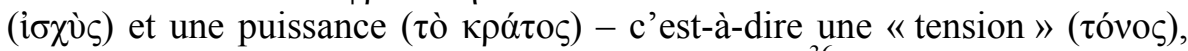

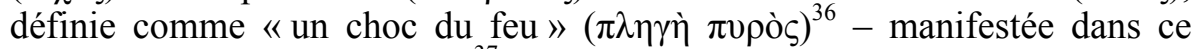

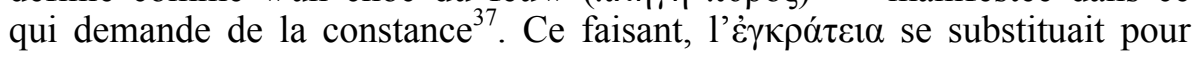

34. D. L. VII, 93 (trad. Goulet).

35. Cf. Stobée, Ecl. II, 60, 9 W. (=SVF III, 264 ; = LS 61 H). J.-B. Gourinat, «Akrasia and enkrateia in ancient Stoicism: minor vice and minor virtue?», dans P. Destrée et C. Bobonich (éds.), Akrasia in Ancient Philosophy, Leyde, Brill, 2007, p. 218, n. 15, suppose que ces définitions sont chrysippéennes et non zénoniennes, Zénon définissant

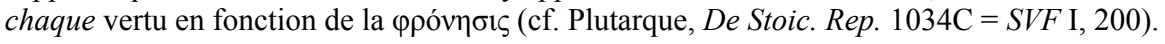

36. Cf. Plutarque, De Stoic. Rep. 1034D6.

37. Ibid. 1034D9-10. 
lui à la $\varphi \rho o ́ v \eta \sigma ı \varsigma^{38}$. Épictète reprend cette idée en identifiant la sagesse et la vertu à la maîtrise de $\operatorname{soi}^{39}$, cette beauté morale dont on fait l'éloge ${ }^{40}$, celle que perd l'homme adultère ${ }^{41}$, celle qui fait l'homme libre ${ }^{42}$ qui plaît aux

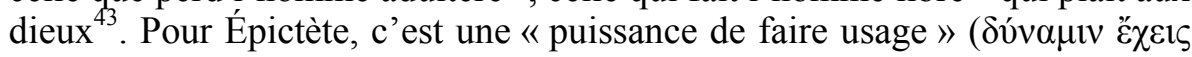

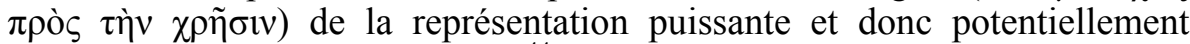

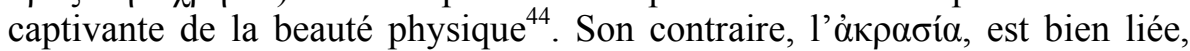
pour Chrysippe, au plaisir, comme en témoigne un passage du $\pi \varepsilon \rho \grave{i} \pi \alpha \theta \tilde{\omega} v$ d'Andronicus ${ }^{45}$. Autrement dit, le fort est celui qui est capable d'interpréter correctement les impressions puissantes qu'il reçoit, comme on le voit dans le phénomène pro-pathologique ${ }^{46}$, là où le faible est captif d'elles du fait de son incapacité à les interpréter correctement.

À l'opposé du puissant Héraclès ${ }^{47}$, on trouvera deux modèles de faiblesse morale ${ }^{48}$, Ménélas, brillant par son incapacité à agir, et Médée, par son incapacité à se contrôler. Reste à savoir si ces derniers peuvent être excusés pour leur faiblesse. Autrement dit, comment Épictète peut-il réduire la faiblesse à un défaut cognitif sans anéantir en même temps la responsabilité morale du faible?

Il serait utile de rappeler qu'Aristote avait déjà donné une réponse à cette question, en considérant l'acrasie comme une disposition par laquelle on commet le mal en connaissance de cause, et non comme une absence

38. Sans qu'elle soit réduite à ses caractéristiques physiologiques - puisqu'il reconnaît que la vertu s'enseigne (cf. D.L. VII, 91) - il insiste néanmoins beaucoup à leur sujet.

39. Cf. Épictète, E. II, 21, 9.

40. Ibid. III, 1, 8-9.

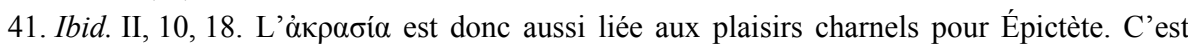

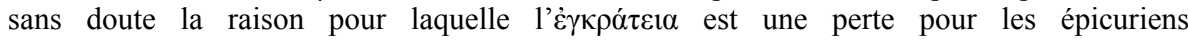
(voir II, 20, 13).

42. Ibid. IV, 1, 9-11.

43. Ibid. I, 13, 1 .

44. Id., M. X.

45. Andronicus, $\pi \varepsilon \rho \grave{i} \pi \alpha \theta \tilde{\omega} v 2,9.5$.

46. Je me permets de renvoyer ici à mon article sur les affections préliminaires, «Deux théories stoïciennes des affections préliminaires », dans la Revue de Philosophie Ancienne, n², 2014, p. 225-257.

47. Cf. Épictète, E. I, 6, 32-36 (Héraclès s'exerce et trouve des occasions dans les circonstances fâcheuses pour révéler ses qualités morales et physiques) ; II, 16, 44-45 (modèle d'Héraclès qui n'est pas resté chez lui mais qui a parcouru le monde) ; II, 18, 22 (Héraclès, modèle pour les athlètes et comparé à Socrate) ; III, 22, 57 (Héraclès est celui qui accomplit avec diligence ce qui lui est demandé) ; 26, 31-32 (Héraclès ne se plaignit pas de ce que Dieu ne lui avait rien donné, mais régna sur terre et sur mer en purifiant les hommes - parallèle en 24,13$)$; IV, 10, 10 (Héraclès affronte les difficultés sans se dérober).

48. Épictète cite Ménélas comme celui qui a considéré la fuite d'une femme adultère, Hélène, comme une perte plutôt que comme un gain (E. I, 28, 12-13), et qui est devenu l'ennemi de son ancien ami Alexandre pour cette raison (II, 22, 23), parce qu'il plaçait son bien dans sa

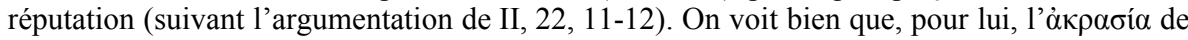
Ménélas n'a rien d'une défaite de la partie rationnelle sous les coups de désirs vigoureux, mais bien plutôt, sous ceux d'une opinion fautive. 
de maitrise de soi. Aux yeux du Stagirite, seules les actions consenties

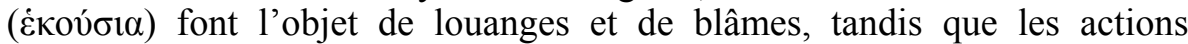
non consenties $(\dot{\alpha} \kappa o v ́ \sigma ı)$ font appel à l'indulgence et à la pitié (définition utile pour récompenser ou châtier les hommes) ${ }^{49}$. Ayant défini l'acte non consenti comme accompli par violence ou par ignorance ${ }^{50}$, Aristote précise

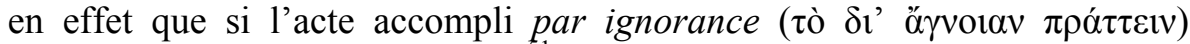

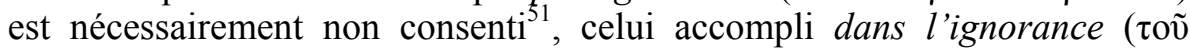

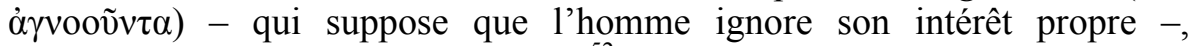
n'implique pas le non-consentement ${ }^{52}$ et le rend donc responsable de sa méchanceté ${ }^{3}$.

Aristote exposera la thèse socratique ${ }^{54}$ pour la réfuter, thèse suivant laquelle l'acrasie n'existe pas $^{55}$ puisqu'on ne peut que choisir le meilleur apparent et parce que c'est toujours contre son gré - et donc «par ignorance $»-$ qu'on se trompe ${ }^{56}$ (thèse qu'adopteront respectivement Epictète et Marc Aurèle) ${ }^{57}$. Or, dans l'argumentation qu'il développe par la suite $^{58}$, Aristote établit contre Socrate la possibilité d'une science inopérante

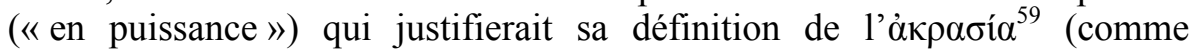
disposition où l'on commet le mal en connaissance de cause), tandis qu'il pense pouvoir sauver sa position en montrant que l'ignorance du faible, qui fonde en définitive sa responsabilité morale, n'est pas située au niveau de la connaissance scientifique mais de la connaissance sensible ${ }^{60}$. Autrement dit, l'acratique accomplirait un acte mauvais auquel il a consenti, qu'il désire, et dont il devient coupable malgré son ignorance (conçue comme un déficit de connaissance sensible). Cela permet à Aristote de retrouver le sens

\section{Cf. Aristote, $E N, 1109 \mathrm{~b} 30-35$.}

50. Id. 1109b35-1110a1.

51. Ibid. $1110 \mathrm{~b} 18$.

52. Ibid. 1110b30-31.

53. Ibid. 1110b31-32. C'est dire avec R. Bodéüs (dans sa traduction de l'EN, p. 136, note 3) que « le méchant n'ignore jamais ce qu'il fait, mais il ignore toujours que ce qu'il fait est mal, précisément parce qu'il est méchant. L'ignorance "responsable de sa méchanceté" est donc ici la perversion totale du jugement qui lui fait toujours prendre le mal qu'il fait pour le bien ».

54. Ibid. 1145b22-27. Socrate conteste ainsi la troisième « opinion recevable » (constitutive

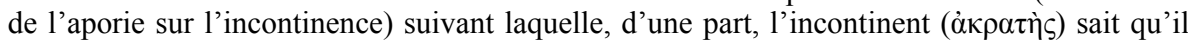
accomplit de mauvaises actions mais n'y peut rien du fait d'une affection qui l'y contraint et,

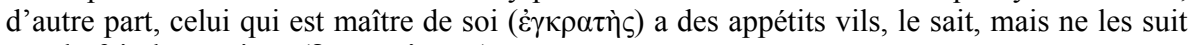

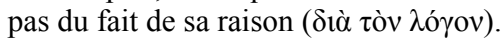

55. Cf. Platon, Protagoras 352d-e.

56. Id. 358c-d ; Gorgias 468c, 509e ; Sophiste 228c-e ; Lois 731c ; République II, 382a-b ; III, 413a ; IV, 443e.

57. Pour la reprise du Timée (86d) chez Épictète, voir E. I, 28, 4 et II, 22, 36. Chez Marc Aurèle, voir $P$. VII, 63. En $E$. II, 26, 1-3, Épictète reprend la thèse platonicienne en lui donnant un fond naturaliste, comme il l'avait fait par ailleurs (par exemple, en $E$. I, 28, 2).

58. Cf. Aristote, EN, 1146b24-1147b19.

59. Id. 1146b31-1147a24.

60. Ibid. 1147b13-17. 


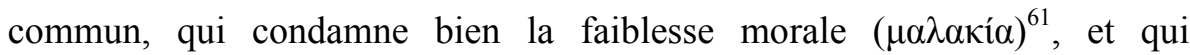
n'entretient aucune espèce d'indulgence pour le méchant qui ne tient pas fermement ses croyances face à ses appétits vigoureux ${ }^{62}$.

Si l'on compare la position aristotélicienne à celle d'Épictète, on remarque une continuité et une rupture. Suivant Aristote, le stoïcien soutient bien que le coupable consent à agir. Cela le rend responsable de fait (puisqu'il est l'unique cause de l'action) et, partant, de droit (puisque cette cause est une opinion à laquelle il s'identifie et non un appétit ou une quelconque manifestation d'une partie irrationnelle en lui). Mais rompant avec le Stagirite, il affirme que le coupable n'a pas cédé devant ses appétits vigoureux «par faiblesse» ni «dans l'ignorance», mais a agi à la fois de manière consentie (par assentiment) et «par ignorance ${ }^{63}$, le caractère « involontaire » de son erreur ${ }^{64}$ témoignant d'un conflit ( $\left.\mu \alpha ́ \chi \eta\right)$ qui implique l'âme tout entière (une contradiction en réalité, puisque l'âme est de part en part rationnelle). Ayant agi nécessairement en vue de l'avantage ( $\sigma u \mu \varphi \varepsilon ́ p o v)$,

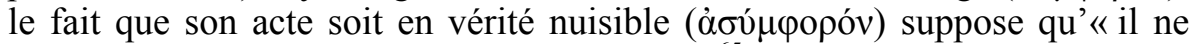

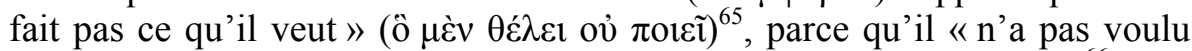

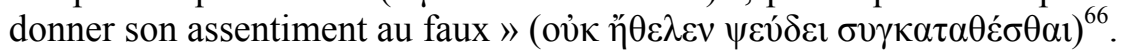

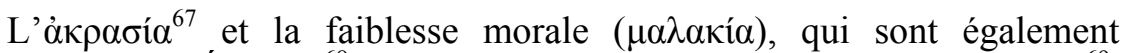
blâmables pour Épictète ${ }^{68}$, doivent donc être «chassées de la pensée » ${ }^{69}$. Cette version rejoint la thèse traditionnelle du Portique, selon laquelle l'intempérance est à l'origine de toutes les passions, ces «maladies de l'âme $\rangle^{70}$ comprises comme autant de défaillances de l'esprit et de la raison droite $^{71}$.

Plus qu'Aristote, le stoïcien insiste sur la responsabilité du faible. Si

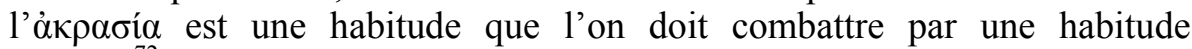
opposée $^{72}$, elle est également réduite à une opinion qui consiste à placer ses

\section{Ibid. $1145 \mathrm{~b} 10$. \\ 62. Ibid. $1146 \mathrm{a} 2-4$.}

63. Stobée, Ecl. II, 88, 8 - 90, 6 (=SVF III, 389 ;= LS 65 A), distingue l'ignorance proprement pathologique, qui ne se dissout pas par des démonstrations. Il y a conflit avec ce que dit Épictète en $E$. II, 26, 4-7 (et en I, 28, 8), sur le fait de « montrer clairement » au fautif qu'il se trompe pour qu'il ne commette plus son erreur.

64. Cf. Épictète, E. I, 17, 14 ; II, 22, 36.

65. Id. II, 26, 1-3.

66. Ibid. I, 28, 4.

67. Ce terme apparaît deux fois chez Épictète, une fois chez Galien (qui cite Chrysippe : PHP IV, 4, 24-25) et quatre fois en général, en D.L. VII, 92-93 (=SVF III, 265) et chez Stobée, Ecl. II, 59, 4-60, 2 ; 60, 9-24 (= LS 61 H).

68. Id. III, 1, 8. En II, 21, 6-7, il montre que les gens trouvent dans l'amour une excuse pour être intempérants, comme s'il s'agissait d'un acte involontaire.

69. Ibid. II, 16, 46.

70. Ibid. 10-11, 24.

71. Cf. Cicéron, Tusc. IV, 10, 22.

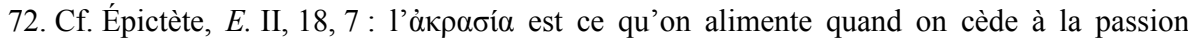

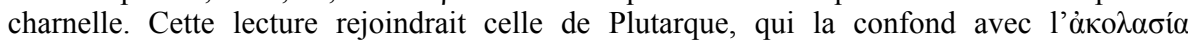


biens et soi-même dans les choses étrangères à sa prohairésis ${ }^{73}$. Par

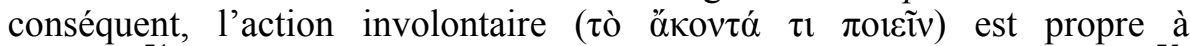
l'esclave $^{74}$, qui désire les choses étrangères ( $\left.\dot{\alpha} \lambda \lambda \operatorname{có}_{\tau \rho} \alpha\right)$ à sa prohairésis ${ }^{75}$, celles qui ne dépendent pas de lui. L'acratique sera celui dont l'attitude est irresponsable, non pas parce qu'il agirait mal en connaissance de cause - comme Aristote le voudrait -, mais au contraire, parce qu'il se fait du mal du fait qu'il ignore la différence entre ce qui dépend de lui (c'est-à-dire sa prohairésis et ses activités propres) et ce qui ne dépend pas de lui.

\section{Ménélas, vaincu par la beauté d'Hélène}

Qu'en est-il à présent du faible incapable d'agir? D'où vient sa faiblesse et est-elle excusable ? Les stoïciens paraissent assumer deux types de résistances à l'action, dont il faut comprendre qu'elles n'entament en rien la responsabilité de l'agent. D'une part, il existe des résistances internes (le

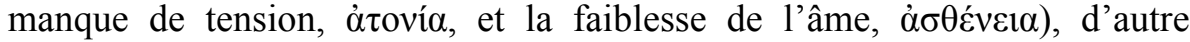
part, on trouve des résistances externes (la faiblesse physique et les empêchements). Si les résistances externes sont largement amoindries par le caractère providentiel de tout événement, sur lequel nous ne reviendrons pas, reste à savoir en quoi consistent les résistances internes, c'est-à-dire ce qui a empêché le faible d'agir. Notre principale source ici n'est pas stoïcienne mais anti-stoïcienne. C'est Galien qui rapporte que la passion, pour Chrysippe, peut avoir pour cause une autre faculté que la faculté rationnelle, à cause du «manque de tension» et de la «faiblesse de l'âme» :

«[Chrysippe] dit qu'il y a des moments où nous nous détournons des décisions correctes parce que la tension de l'âme se relâche et ne persiste pas jusqu'à la fin, ou n'exécute pas complètement les ordres de la raison, et il indique clairement ce qu'est la passion chez les personnes dans cette situation. Je vais désormais citer un passage de Chrysippe qui donne des explications à ce sujet. Il s'agit d'un extrait de la partie éthique de son traité Sur les passions : "C'est en accord aussi avec cela que les tensions concernant le corps sont dites manquant de tension ou pourvues d'une bonne tension en référence à l'ensemble des nerfs, du fait que nous avons de la force ou nous manquons de force dans les actions accomplies au moyen de ces nerfs ; quant à la tension qui est dans l'âme, elle est dite tension au sens de bonne tension ou de manque de tension". Il poursuit: "De même que dans une course ou dans une activité où

(cf. De Virt. Mor. 445B3-5), et celle de Sextus (Adv. Math. IX, 153-154), qui la distingue de l'endurance corporelle ( $\kappa \rho \tau \varepsilon \rho i ́ \alpha)$. Mais chez Plutarque il s'agissait de contrer l'argument

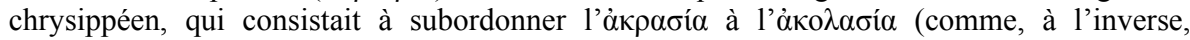

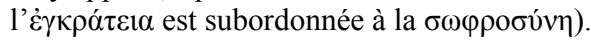

73. Id. II, 22, 28.

74. Ibid. IV, 1, 11.

75. Ibid. IV, 1, 129 ; 10, 29. 
l'on fait preuve de ténacité et dans des actions similaires accomplies à l'aide des nerfs, il y a une manière d'être propice à l'accomplissement de ce qu'on entreprend et une propice au relâchement, quand les nerfs se fatiguent et faiblissent, de façon analogue dans l'âme aussi il y a un état nerveux qui est tel, si bien que nous disons par métaphore que des gens sont sans nerfs ou qu'ils ont du nerf". Expliquant ensuite cela même, il écrit ce qui suit: "L'un recule lorsque surviennent les dangers, un autre s'est relâché et a cédé quand il a reçu une récompense ou une punition, un autre de même dans un bon nombre d'autres situations identiques. Car chacune de ces situations nous met en déroute et nous rend esclaves si bien que, en leur cédant, nous trahissons amis et cités et nous nous prêtons à maintes actions honteuses, une fois que notre premier mouvement s'est relâché. C'est ainsi qu'Euripide a présenté Ménélas. Il sort son épée et se précipite vers Hélène pour la tuer. Mais la voyant, il est frappé par sa beauté et laisse tomber son épée, incapable qu'il est de

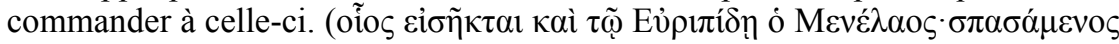

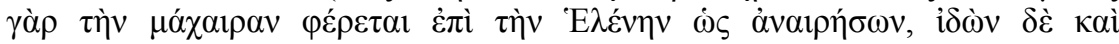

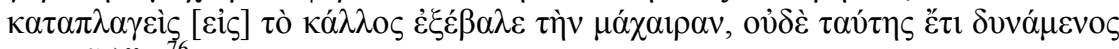

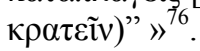

La faiblesse de la tension de l'hégémonique doit être identifiée soit à la faiblesse du jugement $(\tau \tilde{\eta} \varsigma \dot{\alpha} \sigma \theta \varepsilon v o v \tilde{~} \dot{v} \pi \mathrm{o} \lambda \hat{\eta} \psi \varepsilon \omega \varsigma)^{77}$ qui désobéit à la raison " par faiblesse " ${ }^{78}$, soit à une forme de précipitation et d'impatience de l'acte $\mathrm{d}^{\text {'assentiment }}{ }^{79}$. Galien choisit la première solution :

«Si quelqu'un, sous la pression de la colère ou la séduction du plaisir, s'écarte de ses jugements initiaux, son âme est faible, elle manque de tension, et le mouvement de cette âme est la passion ${ }^{80}$.

Marie-Odile Goulet-Cazé interprète ce passage comme suit :

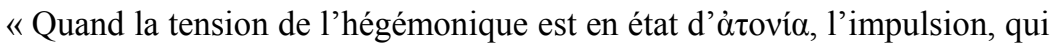

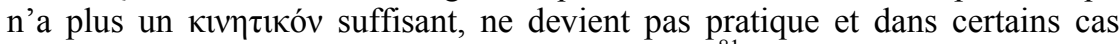
l'hégémonique peut devenir la proie des passions $»^{81}$.

Selon Goulet-Cazé, l'action peut être annulée par manque de tension dans l'hégémonique ${ }^{82}$. Ce faisant, elle fait référence à ces actes manqués

76. Galien, PHP, IV , 6, 1-9 ; p. 270, 21-272, 6 De Lacy (= SVF III 473) (trad. M.-O. GouletCazé).

77. Cf. Stobée, Ecl. II, 7, 10.

78. Cf. Galien, PHP IV, 2, 25, p. 242, 35-36 De Lacy; Clément d'Alexandrie, Strom. II, 13, 59, 6 (= SVF III, 377).

79. Cf. Plutarque, De Virt. Mor. 7, 447A (=SVF III, 459); De Stoic. Rep. 1056E-F (= SVF II, 993 ; = LS $41 \mathrm{E}$ ).

80. Galien, PHP IV, 6, 19 ; p. 274, 5-8 De Lacy (trad. M.-O. Goulet-Cazé).

81. M.-O. Goulet-Cazé (éd.), Études sur la théorie stö̈cienne de l'action, Paris, Vrin, 2011, p. 111.

82. Ibid. p. 107. 
dont parle Alexandre d'Aphrodise ${ }^{83}$. Il s'agit de savoir si le « relâchement » de Ménélas, c'est-à-dire son recul et son renoncement face à Hélène, supposent une baisse de tension de la partie directrice de son âme - là où, au contraire, l'exemple de la course témoigne d'une ténacité et d'une vigueur dans les nerfs. En commentant un passage d'Origène ${ }^{84}$, le même interprète remarque très justement la présence du vocabulaire de la cause, qu'elle rattache à la distinction chrysippéenne :

« La femme séductrice, [dit Origène], n'est pas "une cause qui suffit à lui faire abandonner son intention". Cette femme n'est qu'une "cause prochaine et auxiliaire", pour parler en termes chrysippéens ; en réalité, la 'cause complète et principale', c'est l'état de faiblesse dans lequel se trouve le $\pi v \varepsilon \tilde{v} \mu \alpha$ de l'hégémonique de cet homme et de cet état de faiblesse il est pleinement responsable. [...] Cette interprétation s'harmonise avec l'idée stoïcienne que nous sommes responsables de tous nos actes, même de ceux pour lesquels nous avons tendance à croire que notre responsabilité n'est pas engagée. Les représentations ne sont que des causes auxiliaires, la cause principale tient à l'état de notre hégémonique et à la façon dont nous avons su fortifier et affermir notre raison $»^{85}$.

Dans sa note, Goulet-Cazé explique que la responsabilité de l'homme séduit par la femme tient, selon Origène, au fait qu'il a mal éduqué sa raison. Bien que cette lecture soit astucieuse parce qu'elle fait usage de la théorie chrysippéenne des causes ${ }^{86}$, l'idée d' « acte manqué » par « faiblesse d'une tension de l'hégémonique » relèverait plutôt d'une interprétation antistoïcienne que stoïcienne.

En effet, la faiblesse du jugement de Ménélas prend deux formes différentes selon que l'on considère son acte manqué (tuer Hélène) et son acte accompli (déclenchement de la guerre de Troie). Si Galien évoque l'acte manqué de Ménélas (le meurtre d'Hélène) au moyen de la faiblesse du souffle de l'hégémonique, Épictète veut montrer au contraire que son acte a bien été accompli (dans le déclenchement des hostilités), à cause d'une vision normative ${ }^{87}$ qui l'aurait rendu esclave de ce qui aurait dû au contraire

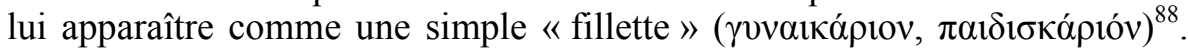
La « faiblesse » de Ménélas qui, dans un cas, lui fait jeter son épée devant la

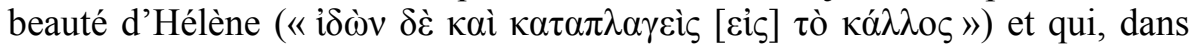
l'autre cas, lui fait déclarer la guerre aux Troyens en se représentant comme un dommage la perte d'une femme adultère, implique nécessairement une interprétation fautive. Autrement dit, l'acte manqué de Ménélas n'est pas

83. Cf. Alexandre d'Aphrodise, De Anima, p. 73, 1-2 Bruns. Goulet-Cazé [2011], p. 109.

84. Origène, De Principiis III 1, 2, 1-5, 26 (= SVF II, 988 ; = LS 53 A).

85. Goulet-Cazé, op. cit., p. 112-3.

86. Bien qu'elle puisse passer pour aristotélicienne, en ce qu'elle fonde la responsabilité sur une possibilité passée. Voir Aristote, EN III, 7, 1114a4-9.

87. Cf. Épictète, E. I, 28, 12-13.

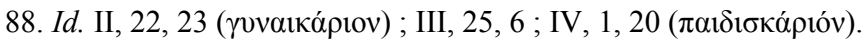


l'expression d'une impuissance dogmatique, d'une perte de la raison (sauf à considérer la volonté de tuer Hélène comme un acte de sagesse...), mais bien d'une interprétation qui le paralyse sur le moment mais qui le fera agir plus tard. Galien interprète donc la faiblesse de l'âme comme une défaillance de la raison, quand Ménélas ne parvient plus à «commander à sa dague »

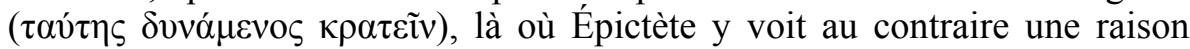
viciée, choquée par ses propres constructions mentales.

Néanmoins, la citation de Chrysippe n'insiste pas tant sur l'impuissance « physique » et sur la mollesse de Ménélas que sur sa faiblesse cognitive, qui lui fait changer d'avis à l'apparition d'Hélène. Autrement dit, Ménélas ne tient pas «fermement» ses résolutions malsaines - ce qui pourrait même passer pour un acte raisonnable, dans la mesure où l'entêtement à les tenir étant au contraire une «absence de tension» ( $\dot{\alpha} \tau$ ovía $\alpha)^{89}$. Dans le cas de Ménélas, il s'agit de cette oscillation pathologique dont parle Plutarque ${ }^{90}$, qui ne contredit pas l'unicité de l'âme ${ }^{91}$, et de cette incohérence face aux représentations ${ }^{92}$. Par conséquent, l'absence de tension correspond à une faiblesse cognitive, et la « baisse de tension de l'hégémonique » ne constitue en rien une explication "physique» de ce phénomène qui excuserait par avance Ménélas. Bien sûr, la beauté « impressionnante » d'Hélène fait d'elle une cause occasionnelle, littéralement physique, pour le faible d'une mauvaise interprétation, mais non sa cause principale. Il faudrait donc plutôt $\mathrm{y}$ voir une description du phénomène pathologique dont Ménélas est le parfait exemple. De même que le sommeil produit, chez celui qui s'endort, un relâchement de la tension pneumatique ${ }^{93}$, de même l'apparition d'Hélène a fait que ce dernier a relâché son arme, parce que sa partie directrice n'était pas assez forte pour y résister ${ }^{94}$.

Pour cette raison, Ménélas se distingue d'Héraclès, mais aussi de Médée. Héraclès, modèle de vertu, et Médée, modèle de vice, auraient tous deux été suffisamment «forts » pour résister à l'apparition de l'aimé. Mais là où Héraclès est moralement fort, la faiblesse morale de Médée donne lieu, au contraire de Ménélas, à un phénomène de surtension rationnelle, qu'avait bien saisi Sénèque ${ }^{95}$, un court-circuit de l'hégémonique - dont la conséquence est observable dans le comportement de Médée - causé par ses productions psychiques. L'absence de tension physique sera donc avant tout morale parce que cognitive ${ }^{96}$, la tension ( $\tau$ óvo $\varsigma$ ) pouvant être l'attribut des sages (Héraclès) comme des vicieux (Médée). Preuve de sa faiblesse,

89. Ibid. II, 15 .

90. Plutarque, De Virt. Mor. 446F1-447A11.

91. Id. 447C9-D1.

92. Cf. Épictète, E. II, 15,$20 ; 22,6$.

93. Cf. D.L. VII, 58.

94. Voir T. Bénatouïl, Faire usage : la pratique du stoïcisme, Paris, Vrin, 2006, p. 173, sur le cas du sommeil et de la perte de la vertu.

95. Voir De Ira II, 2-4.

96. Cf. Épictète, E. II, 15, 3-4. 
Médée, qui semble balancer entre les extrêmes, est proprement incurable lorsqu'une vigueur malsaine s'ajoute à ses atermoiements ${ }^{97}$.

Pour Galien, l'absence de tension et la faiblesse de l'âme contredisent l'assentiment et l'impulsion préalables et imposent, par le biais d'une représentation impulsive ( $\varphi \alpha v \tau \alpha \sigma i ́ \alpha$ ó $\rho \mu \eta \tau \iota \kappa \eta)$, une action contraire aux « résolutions » de Ménélas. Pour Épictète, au contraire, l'apparition d'Hélène ne provoque qu'une nouvelle interprétation, qui "mesure » à son tour une action positive. Autrement dit, Ménélas a posé sa dague parce qu'il a trouvé "plus avantageux » de ne pas tuer Hélène que de la tuer, et non parce qu'il en aurait été incapable à cause d'une "impression impulsive » paralysant son action. En définitive, c'est bien une faiblesse de l'âme (et donc cognitive) qui est responsable du changement de comportement adopté par Ménélas, qui suppose son entière responsabilité.

\section{Médée, vaincue par son courroux}

Comme nous l'avons vu, Médée a la figure d'un exemple classique chez les stoïciens pour définir peut-être leur position par rapport à Platon et à Aristote. Ce faisant, la lecture que fait Galien de la Médée de Chrysippe est certainement déformante ${ }^{98}$ :

«Médée, d'autre part, n'est pas persuadée par la raison de tuer ses enfants ; par contre, en ce qui concerne la raison, elle dit qu'elle comprend qu'elle a l'intention de faire quelque chose de mauvais, mais sa colère est plus forte que ses délibérations; c'est-à-dire que sa passion n'est pas maîtrisée et ne suit pas la raison comme un maître, mais jette les rênes et désobéit au commandement $»^{99}$.

La faiblesse morale de Médée serait due à un conflit psychique entre deux parties de l'âme :

«Elle savait qu'elle faisait quelque chose de terrible en proposant de tuer ses enfants et donc elle hésita. [...] Puis la colère la poussa par force vers les enfants, comme un cheval désobéissant qui a vaincu son maître; et la raison à son tour la retint et l'écarta, puis la colère la tira en sens contraire, puis encore la raison. Par conséquent, poussée à plusieurs reprises, quand Médée cède à la colère, Euripide lui prête ces mots : moi aussi, je sais que j'ai l'intention de faire quelque chose de mauvais, mais la colère maîtrise mes délibérations ${ }^{100}$.

97. Id., II, 15, 19-20.

98. Comme le souligne C. Gill, dans «Galien a-t-il compris la théorie stoïcienne des passions ? », Les Passions antiques et médiévales, Paris, PUF, 2003, p. 145-152.

99. Galien, PHP IV, 2, 27 (trad. Gill).

100. Ibid. III, 3, 14-16 trad. Gill. 
Remarquons que la lecture de Galien n'est pas plus stoïcienne qu'aristotélicienne. Elle n'est pas aristotélicienne, puisqu'en reconnaissant qu'elle agit mal mais qu'elle ne peut rien y faire, Médée ne comprend pas ce qu'elle dit (« je sais bien tout le mal que je vais faire, mais...»), à l'instar de ces acteurs ou de ces ivrognes qui débitent du Empédocle sans rien y comprendre, selon le mot d'Aristote ${ }^{101}$. Ne possédant le savoir qu'en puissance et non en acte, ses formules et ses arguments ne sont nullement l'indice qu'elle est savante ${ }^{102}$. Cela ferait de Médée, aux yeux du Stagirite, un parfait exemple de faiblesse morale, parce qu'elle commet l'infanticide en connaissance de cause ${ }^{103}$ mais « dans l'ignorance » de son intérêt propre (elle est donc volontaire et non involontaire), sa connaissance sensible étant perturbée par un désir qui la fait se méprendre sur la valeur de ce qui lui apparaît. En un sens, et comme nous l'avons déjà souligné, cette lecture est très proche de celle des stoïciens, à la différence fondamentale près que le désir, pour ces derniers, est un produit de la raison. L'infirmité cognitive et l'aveuglement de Médée n'ont donc pas le même sens pour Aristote ${ }^{104}$ que pour Épictète.

\section{L'erreur interprétative de Galien est facilement dissipée grâce à Plutarque :}

«Certains (les stoïciens) disent que la passion n'est pas autre chose que la raison et qu'il n'y a ni dissension ni conflit entre les deux, mais un infléchissement d'une raison unique dans deux directions, infléchissement que nous ne remarquons pas du fait de l'acuité et de la vitesse du changement. Nous ne percevons pas que l'instrument naturel de l'appétit et du regret, ou de la colère et de la peur, est la même partie de l'âme, qui est portée vers le mal par le plaisir, et qui dans son mouvement se ressaisit elle-même de nouveau. Car l'appétit, la colère, la peur et toutes les choses semblables sont des opinions et des jugements corrompus, qui n'apparaissent pas dans une partie seulement de l'âme, mais sont des inclinations, les fléchissements, les assentiments, les impulsions de la faculté directrice tout entière et, d'une manière générale, des activités qui changent rapidement, comme les batailles d'enfants dont la furie et l'intensité sont instables et passagères, étant donné la faiblesse de ces enfants $»^{105}$.

101. Cf. Aristote, EN, VII, 5, 1147a 18-19.

102. On pourrait rapprocher également ce passage de Rhétorique 23, 1400b 9-16, dans lequel Aristote évoque la Médée de Carcinos, qui se défend à partir des erreurs qu'elle a commises, en produisant un enthymème que le Stagirite rapporte à la technique oratoire précédant Théodore.

103. Voir Aristote, Poétique, 14, 1453b30, où Médée est notamment opposée à CEdipe, qui n'a reconnu son lien de parenté qu'après avoir tué son père (il n'a donc pas su qu'il commettait le parricide).

104. Richard Bodéüs ( $E N$, p. 163, note 1) souligne qu'Aristote affirme par là l'inefficacité potentielle de la science (contre Socrate), puisque l'erreur (conçue comme déficit au niveau de la connaissance sensible), qui n'est pas l'effet d'une absence de science, est néanmoins la cause de l'actualité de la science (qui reste donc en puissance). Pour Épictète au contraire, le conflit psychologique est une contradiction logique (E. II, 26). Il n'est donc pas question d'absence ou de présence de la science (Socrate), ou de science en acte ou en puissance (Aristote), mais plutôt d'usage droit ou perverti de la raison.

105. Cf. Plutarque, De Virt. Mor., 446F-447A (= SVF III, 459 ; = LS 65 G) (trad. Brunschwig et Pellegrin). 
Plutarque nous apprend en effet que l'oscillation rapide des jugements contraires que l'on trouve chez les passionnés peut nous persuader à tort de l'existence de deux parties dans l'âme qui se répondent en prenant alternativement le dessus l'une sur l'autre, et ce, jusqu'à la cession finale de la partie rationnelle. C'est, par ailleurs, ce que l'on voit dans la Médée de Sénèque, Médée changeant d'avis à plusieurs reprises et se ravisant (v. 137-149 et 937-945), comme c'est aussi le cas chez Euripide (v. 1040, $1046-1048,1049-1050,1055,1051-52)^{106}$. Jackie Pigeaud offre à cette occasion une explication convaincante au moyen d'une comparaison saisissante, qui n'est pas dépourvue d'une certaine touche kantienne ${ }^{107}$ :

"Chrysippe utilisait ces deux vers comme appui de sa démonstration de l'unité de la passion et du jugement (...). Le triomphe du thumos sur les bouleumata n'est pas le résultat d'un duel, mais le déferlement d'une vague qui emporte avec elle la vague précédente et dépasse la lisière où elle s'était arrêtée. Ces deux vagues n'en sont plus qu'une, et c'est le mouvement même de la mer ${ }^{108}$.

Pour Épictète, Médée n'a évidemment pas agi sous la détermination d'une puissance irrationnelle (son « $\theta v \mu o ́ c »)$, mais a suivi l'avantage apparent (se venger de Jason en satisfaisant son courroux plutôt que de sauver ses enfants) ${ }^{109}$. La "faiblesse de Médée » est donc cognitive, et n'a rien à voir avec l'òk $\rho \alpha \sigma i ́ \alpha$ telle que la conçoit Aristote. Paradoxalement (ce que l'exemple de Médée nous fait comprendre, mais pas celui de Ménélas), cette faiblesse interprétative donne lieu à une violence incroyable ${ }^{110}$. À la différence de Ménélas, l'absence de tension de Médée, qui fait son infirmité, implique une vigueur qui lui fait fermement tenir ses résolutions mauvaises ${ }^{111}$. Épictète n'accorderait donc pas à Médée une dualité psychique comme il pourrait le faire pour Ménélas (dont l'action manque de vigueur), puisque son crime est «l'explosion d'une

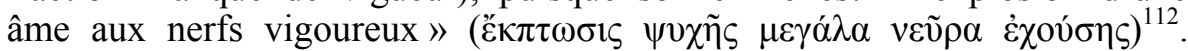
Cette « vigueur», contrairement à ce qui se produit chez un Ménélas aussi mou intellectuellement que moralement, dépendrait même d'une cognition par laquelle, comprenant que ce ses enfants sont qu'il y a de plus cher

106. Sur la comparaison des deux Médée, voir C. Gill, « Two monologues of Self-division : Euripides, Medea 1021-1080 and Seneca, Medea 893-977 », dans P. Hardie, M. Whitby (éds.), Homo Viator : Classical Essays for J. Bramble, Bristol, 1987, p. 25-37.

107. Kant, Anthropologie d'un point de vue pragmatique, III, De la faculté de désirer, $\S 74,252$.

108. J. Pigeaud, La Maladie de l'âme. Étude sur la relation de l'âme au corps dans la tradition médico-philosophique antique, Paris, Les Belles Lettres, 1981, p. 381.

109. Cf. Épictète, E. I, 28, 7.

110. Cf. Galien, PHP IV, 2, 27, p. 244 De Lacy.

111. Cf. Épictète, E. II, 15, 20.

112. Ibid. II, 17, 21. 
à Jason, Médée saisit la meilleure façon de se venger de lui ${ }^{113}$. Bien que négative, cette force interprétative explique ce qui distingue Médée de Ménélas, en donnant à celle-ci un air d'anti-Héraclès qui suscitera quelque part l'admiration d'Épictète ${ }^{114}$.

113. Ce moment est bien décrit par Sénèque dans sa Médée (voir v. 544-551). Ce meurtre, elle le justifie par le destin qu'elle soumet (= v. 520-521 ; parallèle chez Euripide, Méd. v. 10621063 et 1067-1069).

114. Ibid. II, 17, 19. 